\title{
Debridement, antibiotics, and implant retention in infected shoulder arthroplasty caused by Serratia marcescens: a case report
}

\author{
Sungjoon Lim ${ }^{1}$, Jun-Bum Lee ${ }^{2}$, Myoung Yeol Shin ${ }^{1}$, In-Ho Jeon ${ }^{2}$ \\ ${ }^{1}$ Department of Orthopaedic Surgery, National Police Hospital, Seoul, Korea \\ ${ }^{2}$ Department of Orthopaedic Surgery, Asan Medical Center, University of Ulsan College of Medicine, Seoul, Korea
}

\begin{abstract}
Periprosthetic joint infection (PJI) is one of the most devastating complications that can occur after shoulder arthroplasty. Although staged revision arthroplasty is the standard treatment in many cases, surgical intervention with debridement, antibiotics, and implant retention (DAIR) can be an effective option for acute PJI. We report a complex case of infected reverse shoulder arthroplasty (RSA) in a 73-year-old male. The patient had been previously treated for infected nonunion of a proximal humerus fracture caused by methicillin-resistant Staphylococcus epidermidis. He presented with a sinus tract 16 days after the implantation of RSA and was diagnosed with PJI caused by Serratia marcescens. The patient was successfully treated with DAIR and was free of infection at the last follow-up visit at 4 years postoperatively.
\end{abstract}

Keywords: Shoulder; Prosthesis-related infections; Arthroplasty, replacement, shoulder; Debridement; Anti-bacterial agents

Periprosthetic joint infection (PJI) is one of the most dreaded complications after shoulder arthroplasty for both patients and their treating surgeons [1-3]. As the total number of shoulder arthroplasties increases, PJI is becoming a more frequently encountered problem. The goal of PJI treatment is to eradicate the infection while preserving the function of the shoulder. To best control the infection, two-stage revision arthroplasty is generally recommended, especially for chronic PJI. However, the procedure is associated with substantial morbidity, high medical costs, and reduced quality of life. In acute PJI cases, which are defined as occurring within 3 months from the index arthroplasty, less aggressive debridement, antibiotics, and implant retention (DAIR) can be an attractive option. We present an unusual case of infected reverse shoulder arthroplasty (RSA) that was successfully treated with DAIR.

\section{CASE REPORT}

The Institutional Review Board/Ethical Committee of Asan Medical Center determined this project met the conditions for exemption (No. 2021-2264-0001). Obtaining informed consent from the patient was not required in this case report.

A 73-year-old male was referred to our hospital with the chief complaint of pain and weakness in the right shoulder. In 2015, he

Received: September 6, $2021 \quad$ Revised: October 16, $2021 \quad$ Accepted: October 18, 2021

Correspondence to: In-Ho Jeon

Department of Orthopaedic Surgery, Asan Medical Center, University of Ulsan College of Medicine, 86 Asanbyeongwon-gil, Songpa-gu, Seoul 05505, Korea

Tel: +82-2-3010-3896, Fax: +82-2-488-7877, E-mail: jeonchoi@gmail.com, ORCID: https://orcid.org/0000-0002-9289-9193

Financial support: None.

Conflict of interest: None.

Copyright@ 2022 Korean Shoulder and Elbow Society.

This is an Open Access article distributed under the terms of the Creative Commons Attribution Non-Commercial License (http://creativecommons.org/licenses/by-nc/4.0/) which permits unrestricted non-commercial use, distribution, and reproduction in any medium, provided the original work is properly cited. 
had sustained a three-part proximal humerus fracture of the right shoulder during a motor vehicle accident. The patient underwent open reduction internal fixation (ORIF) with a locking plate, which resulted in reduction loss of the greater tuberosity. He underwent revision ORIF 1 month after the initial surgery. Nevertheless, he complained of persistent shoulder pain and loss of shoulder motion postoperatively. Six months after the revision surgery, the patient was diagnosed with nonunion of the fracture at our institution. He had no other underlying medical disease except for hypertension.

The preoperative work-up confirmed a normal erythrocyte sedimentation rate (ESR) and C-reactive protein (CRP) level. The initial surgical plan at our center was revision osteosynthesis using a bone graft. However, pus-like discharge was observed intraoperatively, so the plan was changed to implant removal, debridement, and antibiotic-loaded polymethylmethacrylate bead insertion (Fig. 1). The pus culture was positive for methicillin-resistant Staphylococcus epidermidis (MRSE). After intravenous antibiotic treatment (vancomycin) for 6 weeks, the patient underwent a second debridement surgery. The cement beads were removed, and multiple tissue samples were obtained. All of the tissue cultures were negative.

After confirming that the patient had maintained normal ESR and CRP levels and had been free of any infection signs (fever, wound problems, or bone absorption on radiographs) for 6 months, he underwent RSA (Comprehensive Reverse Shoulder System; Biomet, Warsaw, IN, USA). A standard deltopectoral approach that incorporated the prior incision was utilized. During surgery, we did not observe any pus-like discharge or infected tissue. A frozen biopsy of tissue samples taken during surgery showed no white blood cells per high power field. The rest of the samples were sent for culture. The baseplate was fixed securely
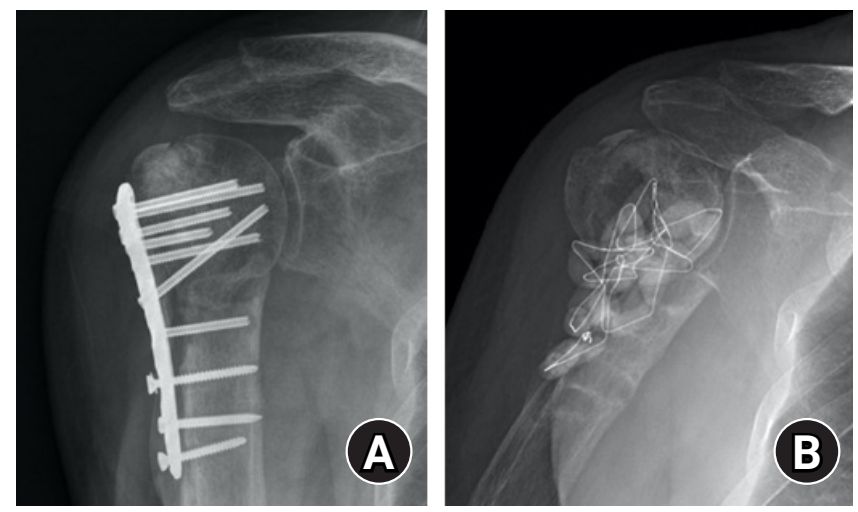

Fig. 1. (A) Anteroposterior (AP) radiograph of the right shoulder showing nonunion of the proximal humerus fracture 6 months after open reduction internal fixation. (B) AP radiograph after debridement and anti-cement bead insertion. with locking screws, and the long humerus stem was fixed with cement (Fig. 2). All procedures were performed without incident.

The patient had an uneventful postoperative recovery. The intraoperative tissue cultures were negative. He was discharged from the hospital on postoperative day (POD) 14 when the postoperative pain control and wound healing were excellent and his CRP level was trending down (from $8.4 \mathrm{mg} / \mathrm{dL}$ on POD 1 to 3.8 $\mathrm{mg} / \mathrm{dL}$ on POD 14). Since this patient had a high risk of infection, an intravenous first-generation cephalosporin antibiotic (cefazolin) was used during his entire hospital stay. He was discharged with instructions to continue an oral antibiotic (cephradine). However, the patient presented with wound dehiscence and a pus-like discharge on POD 16. CRP levels were still elevated $(3.1 \mathrm{mg} / \mathrm{dL})$. Due to the open draining sinus of the surgical wound, the patient was diagnosed with acute PJI. Since he was still in the early postoperative period (approximately 2 weeks from the index arthroplasty), we decided to treat the patient with DAIR. He underwent emergency surgery the next day.

During surgery, we confirmed that pus was draining from the joint (Fig. 3). After tissue samples were acquired, extensive debridement of the infected or nonviable tissue took place. Copious irrigation with pulsatile lavage using diluted povidone-iodine
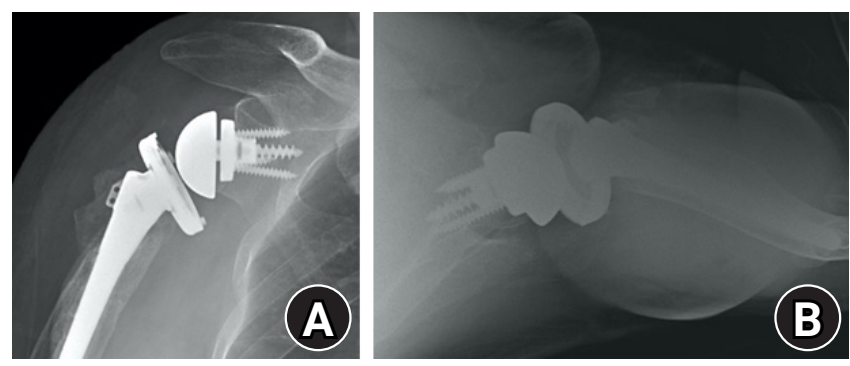

Fig. 2. Plain radiographs (A, B) taken immediately after reverse shoulder arthroplasty.
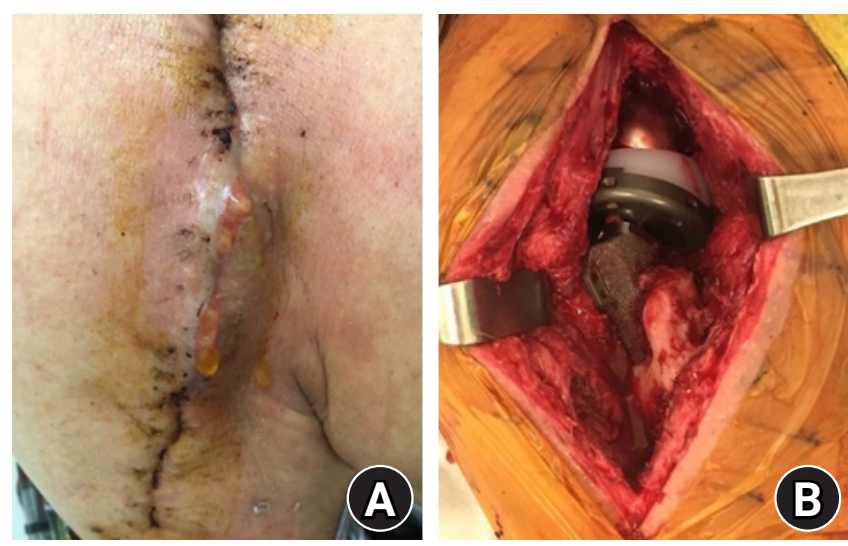

Fig. 3. Intraoperative photographs revealing (A) the draining sinus and $(\mathrm{B})$ a pus-like discharge draining from the joint. 
solution was performed. Because the patient reported postoperative stiffness and osteopenia that likely resulted from having undergone multiple surgeries, we decided not to exchange the indwelling polyethylene liner due to the high risk of periprosthetic fracture that could occur during the dislocation of the components. The wound was closed with absorbable monofilament sutures (PDS II; Ethicon, Cincinnati, OH, USA) over two suction drains.

The tissue cultures were positive for Serratia marcescens in three different samples. An infectious disease specialist was consulted, and an appropriate intravenous antibiotic (ciprofloxacin) was prescribed based on antibiotic susceptibility testing. To treat any residual infection, oral antibiotic therapy (ciprofloxacin) was maintained for 6 months. Since the patient showed good wound healing and normalized ESR and CRP levels, we decided to retain the implant and scheduled regular follow-up visits to monitor his condition.

At the last follow-up appointment 4 years after the final surgery, the wound was clean with no sign of infection, and the patient had maintained normal ESR and CRP levels throughout the follow-up period. Radiographs showed minor scapular notching (Sirveaux grade 1) and no loosening of the stem and baseplate (Fig. 4). We considered the infection to be eradicated as all of the wound aspects, biologic markers (ESR and CRP), and radiographic findings were normal. The patient reported good overall improvement in pain and function throughout the follow-up period. His pain level was tolerable (visual analog scale, 2), and he demonstrated active forward elevation and external rotation of $120^{\circ}$ and $20^{\circ}$, respectively. His functional outcome scores were moderate (American Shoulder and Elbow Surgeons, 60; Con-
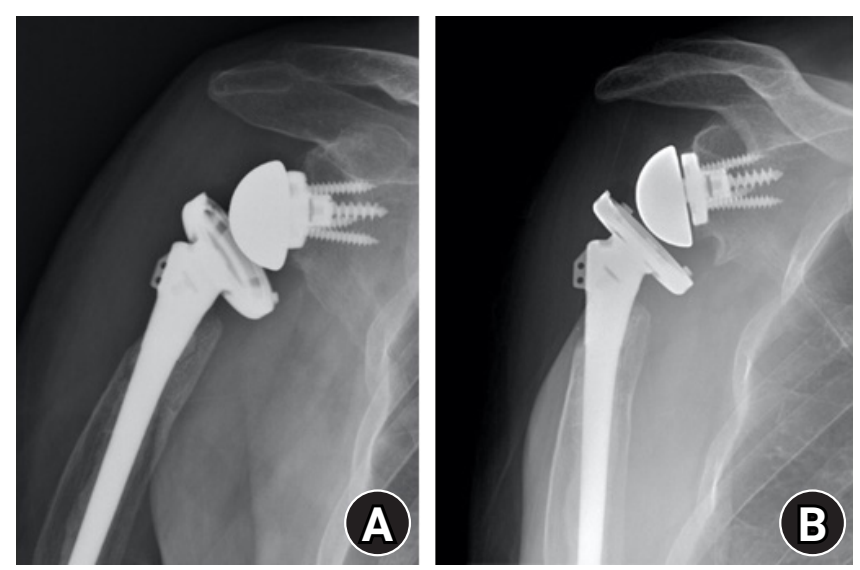

Fig. 4. Postoperative anteroposterior (AP) radiographs (A) obtained immediately after debridement, antibiotics, and implant retention (DAIR) and (B) 4 years after DAIR show Sirveaux grade 1 scapular notching but no evidence of stem or baseplate loosening. stant, 59). The patient was satisfied with the less-aggressive operation to eliminate the infection and accepted the outcome.

\section{DISCUSSION}

RSA was originally developed to overcome cuff tear arthropathy [4]. Recently, the indications for this procedure have expanded to include massive cuff tears without arthropathy and revisions of failed fracture fixation and failed arthroplasty operations [5]. Although RSA has become a viable option for these complex cases, it is not without complications. Of these complications, deep infection is arguably the most devastating and may also affect the outcome. The rate of deep infection after RSA in the literature reportedly ranges from $1 \%$ to $15 \%$; large cohort studies that have included more than 100 patients reported a 3\%-4\% infection rate [6]. Risk factors for PJI after shoulder arthroplasty include diabetes, chronic steroid use, rheumatoid arthritis, and previous surgical procedures [1,7]. In our case, the patient was relatively healthy but had risk factors for infection after RSA, including multiple previous surgeries and a history of infection.

There are a variety of treatment options available for PJI that range from antibiotic suppression, debridement with implant retention, one-stage revision, two-stage revision, and resection arthroplasty $[1,3,7,8]$. Currently, there is no well-defined algorithm to guide treatment for PJI of the shoulder. Therefore, the treatment strategy should be tailored to each patient based on various factors. Above all, the time of presentation after the index arthroplasty is the primary factor. PJI cases can be divided into acute infections ( $<3$ months), subacute infections (3-12 months), and chronic infections ( $>1$ year) [2]. It is generally accepted that debridement without exchanging the implant has a very limited role in subacute or chronic infections $[2,8,9]$. Two-stage revision arthroplasty is more commonly performed in these situations. For acute infections, DAIR has been preferred due to its clear advantages $[3,7,9]$. It is obviously less invasive than staged-revision surgery as it does not involve multiple surgeries or long hospital stays that may cause morbidities. It also minimizes soft tissue damage and prevents bone loss resulting from the explanation of well-fixed implants.

There is also growing evidence obtained from treating PJI in hip/knee arthroplasty patients that DAIR can be an effective treatment option for acute PJI as part of a decision algorithm [9]. The results are mixed across the literature, although studies that follow a decision algorithm generally report infection healing rates $>75 \%$. However, scant data are available about the role of DAIR in treating acute PJI of shoulder arthroplasty. The existing reports are mostly small case series that included varying treat- 
ment protocols. A recent systematic review analyzed the infection outcome by treatment regimen from pooled data [1]. Debridement and implant retention resulted in an infection clearance rate of $68.6 \%$, whereas component revision or resection arthroplasty produced a $>90 \%$ rate of infection clearance. It is therefore logical to assume that if a well-developed decision algorithm is used (as in hip and knee DAIR), the success rate can improve.

The type of pathogen is another important factor for clinicians to consider in these patients. PJI after shoulder arthroplasty is unique in that the most common pathogen is Cutibacterium acnes, followed by coagulase-negative Staphylococcus [3,6]. These are relatively low-virulent organisms and consequently are subject to favorable outcomes. However, antibiotic-resistant organisms, such as methicillin-resistant Staphylococcus aureus, are difficult to eradicate, and treatment with debridement may still result in high failure rates [1,7]. Thus, DAIR is not recommended when antibiotic-resistant strains are confirmed or highly suspected. $S$. marcescens is a ubiquitous, facultatively anaerobic, gram-negative bacillus of the Enterobacteriaceae family [10]. It is an opportunistic pathogen that is responsible for sporadic nosocomial outbreaks and a variety of infections of the musculoskeletal system. Most cases present in immunocompromised populations, such as diabetics. Postoperative infection of the shoulder caused by $S$. marcescens has only been rarely reported in the literature [1].

In the current case, there was an obvious concern for infection after RSA since the patient had previously been treated for chronic MRSE infection. Even though the healthcare providers were attentive to the risk of possible infection during the hospital stay, we were not able to detect the infection. The wound healing was good, and CRP levels were trending down before discharge. It is possible that the continued use of intravenous antibiotics masked the underlying infection until the intravenous administration was discontinued upon discharge. Therefore, treating surgeons should be aware of these potential effects of antibiotics.

It is also important to determine the chronicity of the infection. It was possible that a prior infected nonunion was caused by coinfection with $S$. marcescens and MRSE, but only MRSE was detected. However, since the patient had been infection-free for 6 months after debridement of the infected nonunion and had also demonstrated consecutive negative culture results, we considered the positive results to indicate a newly developed acute infection and treated it accordingly.

We think that our immediate intervention after the onset of symptoms (1 day after wound dehiscence) and the fact that the causative organism was antibiotic-susceptible $S$. marcescens were key factors that led to the satisfactory outcome in this complicat- ed infection. Multidisciplinary care, including our consult with an infectious disease specialist, was crucial. In selected acute infected shoulder arthroplasty cases, DAIR can be an effective treatment option. Further research into the best treatment for acute infections is warranted to determine the role of DAIR and provide further treatment guidelines.

\section{ORCID}

Sungjoon Lim

Myoung Yeol Shin

In-Ho Jeo
Jun-Bum Lee https://orcid.org/0000-0001-6241-2519 https://orcid.org/0000-0002-0202-2671 https://orcid.org/0000-0001-6445-8772 https://orcid.org/0000-0002-9289-9193

\section{REFERENCES}

1. Nelson GN, Davis DE, Namdari S. Outcomes in the treatment of periprosthetic joint infection after shoulder arthroplasty: a systematic review. J Shoulder Elbow Surg 2016;25:1337-45.

2. Sperling JW, Kozak TK, Hanssen AD, Cofield RH. Infection after shoulder arthroplasty. Clin Orthop Relat Res 2001;(382): 206-16.

3. Jacquot A, Sirveaux F, Roche O, Favard L, Clavert P, Molé D. Surgical management of the infected reversed shoulder arthroplasty: a French multicenter study of reoperation in 32 patients. J Shoulder Elbow Surg 2015;24:1713-22.

4. Boileau P, Watkinson DJ, Hatzidakis AM, Balg F. Grammont reverse prosthesis: design, rationale, and biomechanics. J Shoulder Elbow Surg 2005;14(1 Suppl S):147S-161S.

5. Urch E, Dines JS, Dines DM. Emerging indications for reverse shoulder arthroplasty. Instr Course Lect 2016;65:157-69.

6. Zumstein MA, Pinedo M, Old J, Boileau P. Problems, complications, reoperations, and revisions in reverse total shoulder arthroplasty: a systematic review. J Shoulder Elbow Surg 2011; 20:146-57.

7. Boileau P. Complications and revision of reverse total shoulder arthroplasty. Orthop Traumatol Surg Res 2016;102(1 Suppl): S33-43.

8. Assenmacher AT, Alentorn-Geli E, Dennison T, et al. Two-stage reimplantation for the treatment of deep infection after shoulder arthroplasty. J Shoulder Elbow Surg 2017;26:1978-83.

9. Boyer B, Cazorla C. Methods and probability of success after early revision of prosthetic joint infections with debridement, antibiotics and implant retention. Orthop Traumatol Surg Res 2021;107(1S):102774.

10. Mahlen SD. Serratia infections: from military experiments to current practice. Clin Microbiol Rev 2011;24:755-91. 